\title{
La interfaz portugués-castellano en Misiones, Argentina: zona de prueba para la alternancia de lenguas
}

\section{The Castillian-Portuguese interface in Misiones, Argentina: test zone for language alternation}

\author{
JOHN M. LIPSKI \\ Universidad del Estado de Pennsylvania (Penn State), Estados Unidos
}

Este trabajo presenta los resultados de tres experimentos interactivos realizados entre hablantes bilingües (castellano-portugués) en la provincia argentina de Misiones, con la finalidad de investigar si algunas de las restricciones sintácticas postuladas para los cambios de código intra-oracionales son válidas para lenguas tan similares. Los contextos claves para el cambio de idioma son: después de pronombres de sujeto, negativos e interrogativos, y entre verbo auxiliar e infinitivo. Los resultados sugieren una correlación entre la permeabilidad parcial de restricciones sintácticas y distancia genealógica.

Palabras clave: bilingüismo, portugués, alternancia delenguas, cambio de código, psicolingüística

This article presents the results of three interactive experiments carried out among bilingual speakers (Castillian-Portuguese) in the Argentinian province of Misiones, in order to investigate if some of the syntactic restrictions postulated for the intra-sentence code switching are also valid for so similar languages. The key contexts for language switching are: after subject pronouns, negative and interrogative, and between auxiliary verb and infinitive. The results suggest a correlation between partial permeability of syntactic restrictions and genealogical distance.

Keywords: bilingualism, Portuguese, language alternation, code switching, psycholinguistics 


\section{INTRODUCCIÓN}

En las comunidades de habla bilingües, es frecuente la alternancia de lenguas ("code-switching" o cambio de código) dentro del mismo discurso. Cuando el cambio de lengua ocurre dentro de la misma oración (cambio intra-oracional), el hecho de que algunas combinaciones sean poco frecuentes o inexistentes puede señalar restricciones sobre el empleo de dos lenguas en la misma estructura oracional ${ }^{1}$. A pesar de la gran cantidad de entornos bilingües que han aportado datos sobre la alternancia de lenguas, continúa el debate sobre la naturaleza de las circunstancias que desfavorecen los cambios intra-oracionales. Dentro de la gramática generativa se ha postulado que los cambios intra-oracionales requieren una relación apropiada de reacción alrededor de la transición (DiSciullo, Muysken y Singh 1986; Belazi, Rubin y Toribio 1994). Otros investigadores han postulado la imposibilidad de permutar elementos que pertenecen a clases o categorías cerradas como pronombres, preposiciones, determinantes (Azuma y Meijer 1997; Doron 1983; Joshi 1985; Prince y Pintzuk 2000). Asimismo se han implicado los morfemas sistémicos (Myers-Scotton 1997) y la distinción entre categorías léxicas y funcionales (Chan 2008), y en los modelos minimalistas las discrepancias entre los rasgos léxicos (Jake, Myers-Scotton y Gros 2002; MacSwan 1999, 2000, 2004, 2005; van Gelderen y MacSwan 2008; pero véase Hebblethwaite 2007). Otra sugerencia es la optimización de restricciones mutuamente competitivas (Muysken 2013; Bhatt 1997; Hogeweg 2009). La diversidad de estudios y propuestas refleja la complejidad del estudio de las interacciones bilingües, así como la necesidad de complementar las observaciones del habla espontánea con datos replicables. El trabajo que se presenta en los siguientes apartados incluye datos obtenidos en una comunidad de habla donde el portugués y el español están en contacto en un entorno sociolingüístico que facilita la compenetración de las lenguas y que permite poner a prueba algunas de las propuestas sobre los mecanismos morfosintácticos que rigen los cambios de código.

\section{Algunas Restricciones Propuestas SObRE los CAMbios INTRA-ORACiONALES}

Como resultado del análisis de alternancia de lenguas, sobresalen ciertas combinaciones que parecen impedir los cambios de lengua intra-oracionales, en una variedad de entornos bilingües, y que pueden reflejar características (casi) universales sobre la morfosintaxis de las oraciones mixtas. Tomando como prototipo el bilingüismo espańolinglés (en los Estados Unidos y también en Gibraltar; Moyer 1992), se ha observado

\footnotetext{
${ }^{1}$ Por ejemplo, Dussias (2003), Lipski (1977, 1978, 1982, 1985, 2014, 2016b), Toribio (2001a, 2001b), MacSwan (1999, 2000, 2004, 2005), y los trabajos en Bullock y Toribio (2009). Para otros ejemplos de la alternancia entre el castellano y el portugués, véase Aguazo y von Borstel $(2005)$, von Borstel $(2011,2011)$ y Semino y Escobar (2011).
} 
que los cambios de código no suelen producirse después de palabras interrogativas (p. ej., Ebert 2014: 192-198; Peñalosa 1980: 58; Woolford 1983: 531; 1984), entre un verbo modal o auxiliar y un infinitivo (Muysken 2000: 12-13; Peñalosa 1980: 65; Timm 1975: 478-479), entre un elemento negativo y el verbo (Timm 1975: 479), entre clíticos pronominales y verbos (Pfaff 1979), y después de un pronombre de sujeto (Gumperz 1977: 26; Koronkiewicz 2014: Timm 1975: 477). Algunas de estas restricciones aparentes provienen de diferencias sintácticas fundamentales entre el inglés y el español: por ejemplo, el espańol permite sujetos nulos, mientras que el inglés requiere sujetos patentes; los interrogativos y negativos en inglés suelen requerir el apoyo del verbo auxiliar $d o$, etc. Algunos investigadores han propuesto que los cambios intra-oracionales solo pueden ocurrir cuando las estructuras gramaticales de las dos lenguas son congruentes, tanto antes como después del cambio (Poplack 1980; Lipski 1977, 1978, 1982, 1985); por lo tanto, no está claro si las restricciones postuladas responden a características universales de los pronombres, interrogativos, negativos, etc., o si son consecuencias de la falta de compatibilidad morfosintáctica entre determinadas lenguas (p. ej., Clyne 1987; Mahootian y Santorini 1996). En una investigación preliminar, para determinar si las restricciones postuladas sobreviven en la ausencia de discrepancias gramaticales, Lipski (2016a) realizó experimentos psicolingüísticos entre hablantes bilingües en Ecuador, cuyas lenguas eran quichua y chaupi lengua (media lengua), una lengua mixta con morfosintaxis del quichua pero con casi todas las raíces derivadas del español, incluso los pronombres, interrogativos y negativos (Muysken 1979, 1981; Gómez Rendón 2008). Ya que la única diferencia entre las dos lenguas es el origen etimológico de las raíces léxicas, cualquier restricción sobre los cambios de código intra-oracionales no puede ser una consecuencia de discrepancias estructurales. Los experimentos revelaron que, aún en la ausencia de incompatibilidades gramaticales, los cambios de lengua después de pronombres e interrogativos eran desfavorecidos, lo cual sugiere la posibilidad de separar la morfosintaxis y el léxico para estudiar la alternancia de lenguas.

El trabajo que se presenta a continuación ofrece datos de otra configuración bilingüe, que permite el estudio de la alternancia de códigos entre lenguas aun más similares: el español y el portugués. Después de documentar, en el portugués hablado como segunda lengua por personas de habla espańola, la presencia de combinaciones que aparentemente violan las postuladas restricciones, se presentan los resultados de tres experimentos interactivos realizados con participantes bilingües en la provincia argentina de Misiones, donde se hablan los dos idiomas en un entorno sociolingüístico casi completamente libre de presiones prescriptivistas. A pesar de la imagen popular del portuñol como verdadero idioma vernacular de las chacras misioneras, los resultados de los experimentos revelan que los perímetros psicolingüísticos alrededor de las dos lenguas se mantienen intactos, y aun entre dos lenguas tan similares existen algunas combinaciones morfosintácticas desfavorecidas. 


\section{Mezcla de lenguas en el portugués COMo L2}

A lo largo de la frontera de Brasil con los países hispanoparlantes se producen contactos lingüísticos entre el castellano y el portugués. En la mayoría de los casos, los residentes de países hispanoparlantes solo emplean el portugués con interlocutores brasileños, sobre todo los que cruzan la frontera para satisfacer necesidades inmediatas de comercio o gestiones administrativas. Pocos hispanoparlantes en las ciudades fronterizas dominan completamente la lengua portuguesa a menos que hayan tenido una experiencia extensa a causa de estadías en Brasil, estudios formales o matrimonio. Hay dos regiones excepcionales, donde hay comunidades de habla portuguesa dentro de naciones hispanoparlantes. En el norte del Uruguay -en una franja que se extiende bastante lejos de la frontera brasileñase hablan "dialectos portugueses del Uruguay (DPU)", conocidos popularmente como portuñol aunque la mayoría de las estructuras gramaticales provienen del portugués (Elizaincín 1973, 1976, 1979, 1992; Hensey 1972, 1982a, 1982b; Elizaincín, Behares y Barrios 1987; Carvalho 2003a, 2003b, 2004a, 2004b; Douglas 2004; Rona 1960, 1969). El otro caso se encuentra en la provincia argentina de Misiones, según se explicará en los siguientes apartados.

Como preámbulo al análisis del bilingüismo castellano-portugués en Misiones, se analizaron muestras del portugués hablado como segunda lengua, recogidas por el autor en las siguientes comunidades fronterizas (véase el Mapa 1): Paso de los Libres (Argentina); Pedro Juan Caballero, Capitán Bado y Bella Vista Norte (Paraguay); Chuy (Uruguay); Cobija, Guayaramerín y Bolpebra (Bolivia); Ińapari y Santa Rosa (Perú); Leticia (Colombia) y Santa Elena de Uairén (Venezuela) ${ }^{2}$. Los análisis se presentan en Lipski (2008, 2009, 2011a, 2011b, 2014; véase también Kaufman 2009).

\footnotetext{
${ }^{2}$ Agradezco la colaboración de las siguientes personas en la recogida de datos: Ingard Miauchi (Cobija); Kelly Gamboa (Guayaramerín); Derlis Torres (Pedro Juan Caballero, Zanja Pytá, Capitán Bado, Bella Vista Norte); María Silvia Chichizola de Ezama (Paso de los Libres); Pedro González Segura (Leticia); Celso Curi Paucarmaita, Alberto Cardozo, César Ochoa, Jorge Quispe, Narciso Paricahua (Iñapari); Celia Cisneros y Elba Wolf (Santa Elena de Uairén); Graciela Barrios, Selva Chiricó, Adolfo Elizaincín y Magdalena Coll (Rivera); Lidia Vidal, Raquel Puig, Mirta Costa Fernández, Alice Lucas y María Irene Moyna (Chuy).
} 
Mapa 1: Comunidades fronterizas estudiadas

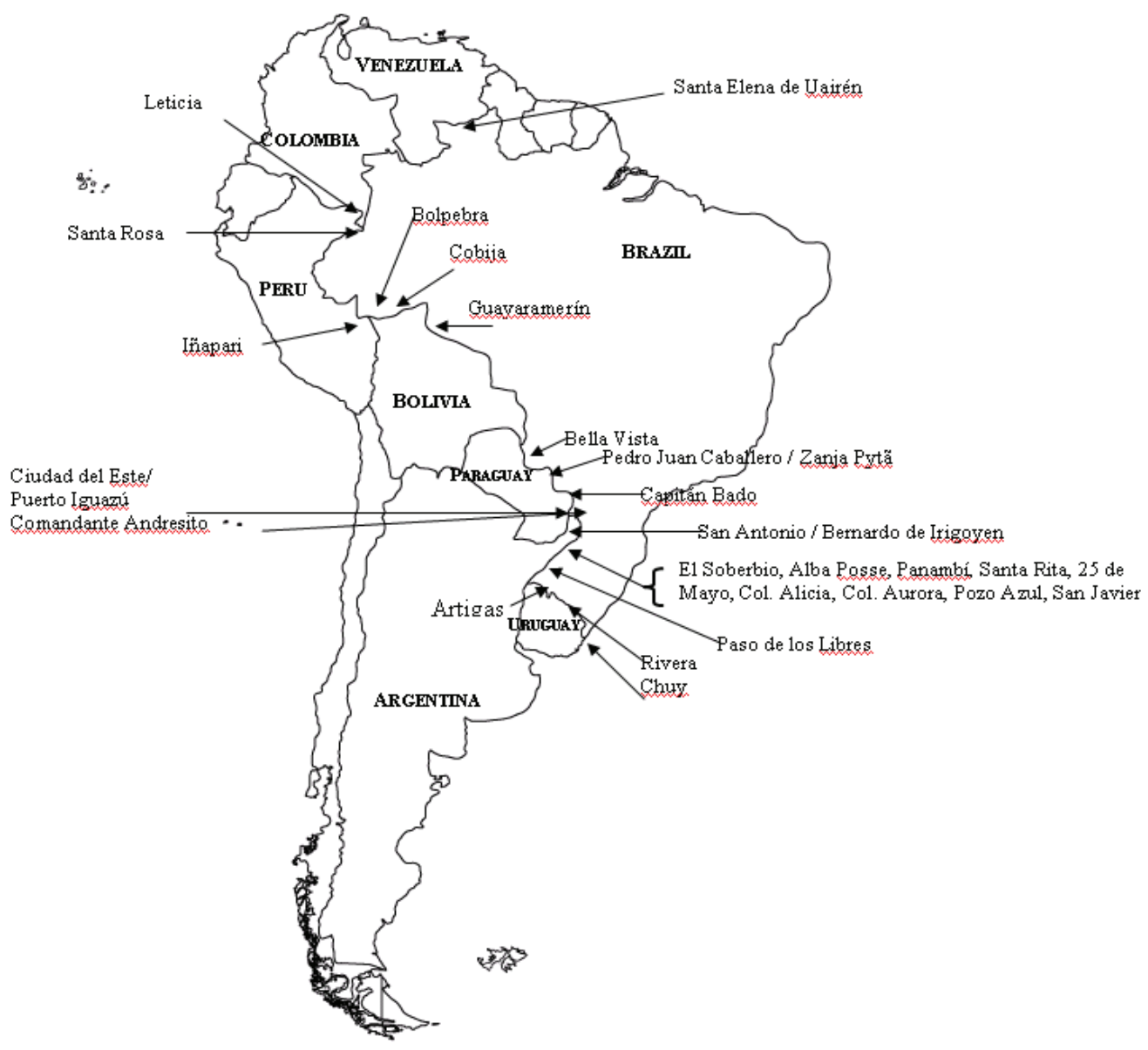

En estas comunidades es posible encontrar algunas combinaciones mixtas que surgen cuando personas de habla española intentan hablar en portugués, por ejemplo con un interlocutor brasileño. Si se tratara de la verdadera alternancia de lenguas entre personas que dominan las dos lenguas, algunas de las combinaciones que ocurren espontáneamente en el portugués hablado como L2 representarían violaciones de las restricciones sintácticas mencionadas anteriormente, pero dado el alto grado de inteligibilidad mutua entre las dos lenguas en zonas fronterizas, las combinaciones mixtas pueden pasar desapercibidas. Algunos contextos en que los hablantes de portugués como L2 han producido combinaciones señaladas como anómalas en algunas investigaciones son: 
ENTRE UN SUJETO PRONOMINAL Y EL PREDICADO:

sei lá yo (Cobija, Bolivia)

ellos ja misturam (Guayaramerín, Bolivia)

eu memo tengo gente que mora lá em Brasil (Guayaramerín, Bolivia)

ello fala direito (Pedro Juan Caballero, Paraguay)

Ella mora aquí (Capitán Bado, Paraguay)

eu puedo trabalhar lá e trabalhar acá (Leticia, Colombia)

yo fala así ticuna no (Leticia, Colombia)

eu tenia más antes (Iñapari, Perú)

ellos falam em Bolivia é mais barato (Iñapari, Perú)

êles tienen que saber (Ińapari, Perú)

todo lo que você dice aquí hay (Ińapari, Perú)

noi hemos precisado de aprender un poquinho (Santa Elena de Uairén, Venezuela)

ENTRE EL VERBO AUXILIAR Y EL VERBO PRINCIPAL:

vine a morar a Corrientes (Paso de los Libres, Argentina)

porque não tem, como le puedo falar, vitrina (Guayaramerín, Bolivia)

o brasileiro que vem hacer compra Ciudad del Este (Pedro Juan Caballero, Paraguay)

não quieren falar a língua di nois (Capitán Bado, Paraguay)

eu puedo trabalhar lá e trabalhar acá (Leticia, Colombia)

tú puedes trabalhar, saca tu CPF (Leticia, Colombia)

ENTRE EL ELEMENTO NEGATIVO Y EL VERBO:

não sabría decirle (Paso de los Libres, Argentina)

¿mas vai ou no vai? (Cobija, Bolivia)

él ja no pode mais (Pedro Juan Caballero, Paraguay)

no hay, no tem portugués (Capitán Bado, Paraguay)

não quieren falar a língua di nois (Capitán Bado, Paraguay)

loh hombre tambem asistem mais os homen não quieren falar (Capitán Bado, Paraguay)

Tabatinga é difíl o turismo porque não tiene loja de artesanato (Leticia, Colombia)

não hay nada pa este povo (Iñapari, Perú)

DESPUÉS DE UNA PALABRA INTERROGATIVA:

quién quer ter mah conocimiento? (Cobija, Bolivia)

você que cosa quer? (Iñapari, Perú)

mozo onde queda Boacha? (Santa Elena de Uairén, Venezuela)

Las alternancias de lengua, inconscientes e involuntarias, producidas por personas que intentan hablar en una lengua que no dominan por completo pueden resultar en combinaciones insólitas sin que esto signifique que sean aceptables entre bilingües equilibrados (Lipski 2008, 2009, 2014, 2016b). En las siguientes secciones se presentan 
datos obtenidos en la provincia argentina de Misiones, donde existen comunidades de habla bilingües (castellano-portugués), que permiten explorar las condiciones gramaticales que afectan la compenetración de las lenguas.

\section{Misiones, Argentina y SU Diversidad lingüística}

La provincia argentina de Misiones es una estrecha península en el extremo nororiental del país, entre Paraguay y Brasil. El Río Paraná forma una frontera natural con Paraguay al oeste; al norte el Río Iguazú separa Misiones de Brasil, y a lo largo de casi toda la frontera oriental de la provincia, el Río Uruguay divide el territorio argentino de Brasil. Misiones llegó a ser territorio oficial de la Argentina en 1881, y a partir de 1883, el gobierno argentino inició una campańa de reclutamiento de inmigrantes europeos, principalmente polacos y ucranianos, para poblar esta región remota y para salvaguardar la soberanía nacional. Después de la Primera Guerra Mundial llegaron muchos colonos alemanes, atraídos por empresas alemanas establecidas en Sudamérica. En 1953, Misiones pasó a ser provincia nacional y sigue recibiendo inmigrantes de países asiáticos y también de los países vecinos.

En Misiones todavía existen núcleos poblacionales que hablan polaco, ucraniano y sobre todo alemán, pero estas lenguas no se transmiten a las generaciones más jóvenes y han de desaparecer del horizonte lingüístico dentro de una o dos generaciones. En algunas comunidades indígenas se habla el mbyá (guaraní) como lengua del hogar. En la franja oriental de la provincia, que se extiende desde las comunidades fronterizas hasta casi el centro de la provincia, se habla el portugués como lengua nativa entre amplios sectores de la población rural. El portugués como segunda lengua circula en grado menor en las mismas comunidades, principalmente entre personas que mantienen vínculos comerciales con Brasil o que tienen contacto diario con obreros hablantes de portugués. La zona de Misiones donde se habla el portugués se caracteriza por una intensa explotación agroforestal, siendo los productos más importantes yerba mate, té, tabaco, caña de azúcar (San Javier), citronela (El Soberbio) y madera. El hilo común que vincula todas las comunidades argentinas de la franja fronteriza misionera es el empleo de la lengua portuguesa como lengua de preferencia entre muchos individuos nacidos y criados dentro del territorio argentino.

La investigadora brasileña Sturza (2005) ha observado que "A língua portuguesa além das fronteiras brasileiras é uma outra língua”, y la interfaz portugués-castellano en Misiones ofrece un caso ejemplar. En uno de los primeros trabajos sobre el tema, Amable (1975: 24-25) caracteriza el empleo del portugués en Misiones como "un verdadero dialecto dentro del habla regional" y comenta que en la franja oriental de la provincia se hablaba solo portugués hasta hace muy poco, y que todavía predominaba el bilingüismo. Daviña (2003) da cuenta de la presencia del portugués y el lenguaje mixto en la literatura y la cultura popular de Misiones, y Maia (2005) ofrece datos pormenorizados sobre el portugués vernacular de Misiones, tomando como punto de partida el habla de alumnos y profesores 
de portugués. Este trabajo documenta las diferencias significativas con respecto al portugués brasileño culto, así como los dominios de uso del portugués dentro de Misiones. En Lipski $(2015,2017)$, se presentan datos actualizados sobre la variabilidad del portugués vernacular de Misiones. Los datos fueron recogidos en las siguientes comunidades misioneras entre 2008 y 2016 (véase el Mapa 2): Puerto Iguazú, Comandante Andresito, San Antonio, Bernardo de Irigoyen, El Soberbio y Barrerinho (General Güemes), Alba Posse, Santa Rita y San Francisco, Colonia Alicia, Colonia Aurora, 25 de Mayo, Panambí, Pozo Azul y San Javier. La mayoría de estas comunidades consiste en un pequeño núcleo urbano y extensas colonias agrícolas pobladas por residentes permanentes y trabajadores, en su gran mayoría nacidos en territorio argentino. Con excepción de San Javier, las comunidades arriba mencionadas tuvieron su origen en el siglo XX. ${ }^{3}$ Para determinar si los ejemplos recogidos entre los hablantes de portugués como segunda lengua son representativos del bilingüismo estable y, por lo tanto, representan posibles excepciones a las postuladas restricciones sobre la alternancia de códigos, se realizaron posteriormente tres experimentos con participantes bilingües en Misiones.

\footnotetext{
${ }^{3}$ Entre las muchas personas que colaboraron con mis investigaciones sobre el portugués misionero, merecen un agradecimiento especial los siguientes individuos: Liliam Prytz Nilsson (Ministerio de Educación, Posadas), Viviana Eich, Ana Zanotti, Liliana Daviña e Ivene Carissini da Maia (Universidad Nacional de Misiones, Posadas), Fabián Ramallo (Posadas), Sergio Chajkowski y Rosendo Fuchs (Panambí), Hugo Cámara Robles (Comandante Andresito), Sandra Grade (Puerto Iguazú), Nélida Aguerre (Alba Posse), Norma Ramírez, Justino Steinhaus y Ricardo Leiva (El Soberbio), Oscar Almeida (Barrerinho), Darío Miranda y Manglio Vargas (Colonia Alicia), Carlos Stikierski (Colonia Aurora), Elsa Rodríguez de Olivera (25 de Mayo), Daniel Ziemann y Carlos Knoll (Santa Rita), Marcelo Ragotín, Cristina Barchuk y Luis Alberto Vogeli (Pozo Azul), Fátima Zaragoza y Juan Carlos Morínigo (Bernardo de Irigoyen), Isabelino Fonseca (San Antonio), Roberto Pinto (San Javier).
} 
Mapa 2: Comunidades estudiadas en la provincia de Misiones, Argentina

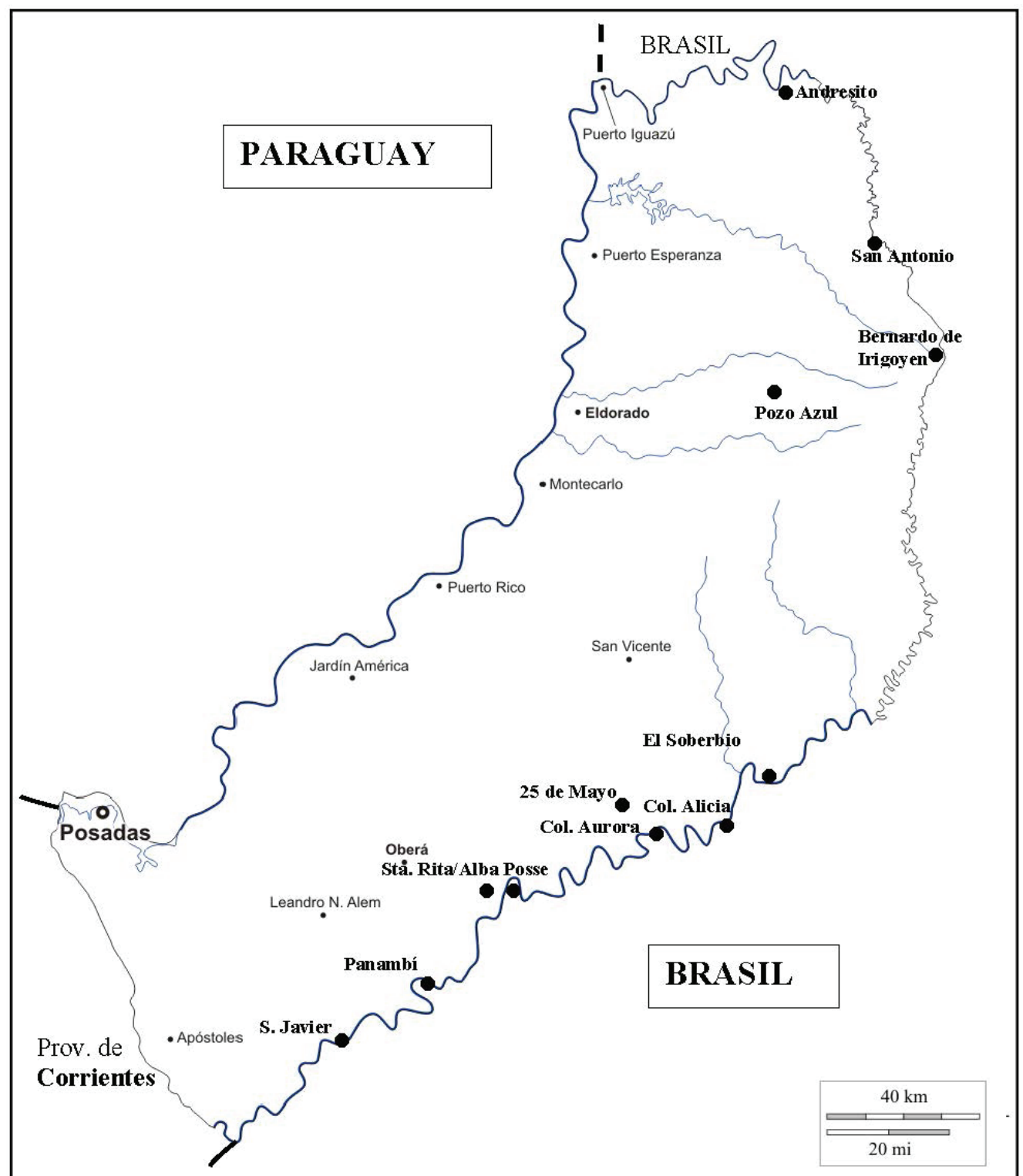




\section{Primer EXPERIMENTO: TRADUCCIÓN RÁPIDA}

El primer experimento empleaba la técnica de traducción rápida entre el español y el portugués, para medir el grado de compenetración de las dos lenguas en contacto en un contexto de priming (preactivación o primado) aumentado. Al mismo tiempo, el experimento servía como sondeo indirecto sobre la identificación de lenguas vernaculares y el grado de aceptabilidad de oraciones mixtas. El experimento se llevó a cabo en El Soberbio y Colonia Alicia.

\section{Participantes}

Participaron cincuenta personas (edades 18-45) de El Soberbio, Colonia Alicia, Colonia Aurora, y Pozo Azul, y las colonias de El Fisco, Barrerinho, Paraíso, y Puerto Londero. Todos hablaban portugués como lengua del hogar (algunos también hablaban alemán); algunos habían adquirido el español en el seno de la familia, y otros no hablaban español hasta que comenzaban la escuela (todos habían alcanzado por lo menos el primer año de educación secundaria). Ninguno de los participantes había vivido en Brasil ni había recibido clases de lengua portuguesa.

\section{Materiales}

Para realizar el experimento, se crearon noventa enunciados por medio de varios programas de texto a voz (lector de texto) en portugués brasileño y español, empleando siempre voces femeninas. Treinta de los enunciados estaban en español, treinta en portugués, y treinta contenían varias combinaciones de español y portugués en la misma oración. Para aproximar la fonotáctica del portugués vernacular hablado en Misiones se realizaba el fonema $/ K /$ como [j] y se eliminaba la / $/$ f final de palabra: así por ejemplo trabalhar [tra.ba.Káx] 'trabajar' se convertía en [tra.ba.já]. Al mismo tiempo se efectuaban unas modificaciones morfosintácticas que prevalecen en el portugués vernacular misionero: se marcaba la /s/ solo en el primer elemento de los sintagmas nominales plurales (p. e. os guri 'los niños'); se suprimía la /s/ final del sufijo verbal/-mos/ de primera persona plural, y para los verbos de la primera conjugación (en -ar) se convertía la vocal temática en /e/: falemo (falamos) 'hablamos', plantemo (plantamos), etc. Para crear los estímulos mixtos era más factible "engańar" los programas de voces portuguesas para aproximar los fragmentos en español, utilizando varias estrategias ortográficas (por ejemplo para lograr nosotros se escribía "nossôtros", para dónde se escribía "dôndei", ellos era "êlhous", etc.). Se ajustaban los detalles segmentales y suprasegmentales con la ayuda del programa PRAAT (Boersma y Weenink 1999-2005) para lograr la mayor consistencia. Cada estímulo consistía en un enunciado, un silencio de $500 \mathrm{~ms}$. y un tono audible. Se presentaba los estímulos de forma aleatoria. Unos ejemplos son:

ENTRE VERBO AUXILIAR E INFINITIVO: que eu saiba os uruguaio no pueden falar guaraní 
ENTRE PALABRA INTERROGATIVA Y PREDICADO

¿Quién quer estudar hoje na tarde?

¿Dónde fica a casa do prefeito?

ENTRE PRONOMBRE DE SUJETO Y VERBO

eu tenho que contá y voh tein que escondé e o que conta tein que achá

EMPIEZA EN ESPAÑOL Y CAMBIA AL PORTUGUÉS

yo nací en Brasil pero vine a morar aquí fais cuatro ano

cuando ellos hablan misturam as língua

EMPIEZA EN PORTUGUÉS Y CAMBIA AL ESPAÑOL

na nossa zona la vida es muy cara

alguns brasileiros entendem lo que nosotros hablamo

\section{Método}

Las instrucciones indicaban que si el participante oía una oración en español (“castellano") debía traducirla al portugués ("brasilero") lo más rápidamente posible, y si escuchaba una oración en portugués debía traducirla al español. No se les revelaba a los participantes que algunas de las oraciones contenían elementos de ambas lenguas. Los participantes escuchaban los estímulos por medio de audífonos y sus respuestas fueron grabadas digitalmente. No había posibilidad de repetición y, si no respondían dentro de dos segundos, se pasaba al próximo estímulo.

\section{Resultados y discusión}

Los resultados de la traducción de oraciones, enteramente en español o portugués, se presentan en Lipski (2017). En general, se notaba poca compenetración de rasgos fonéticos y morfosintácticos. En el cuadro 1 se presentan los resultados de la traducción de las oraciones mixtas. 
Cuadro 1: Resultados de la traducción de enunciados mixtos español-portugués $(\mathrm{N}=50)$

\begin{tabular}{|c|c|c|c|}
\hline & \% >> Espańol & $\%$ >> Portugués & $\%>>$ mixto \\
\hline Español >> Portugués $\{$ todos\} & $70.7 \%$ & $26.8 \%$ & $2.5 \%$ \\
\hline Esp. >> Ptg. $\{$ PRO + $\}$ & $79.4 \%$ & $19.8 \%$ & $0.8 \%$ \\
\hline Esp. $>>$ Ptg. $\{$ aux + infinitivo $\}$ & $91.0 \%$ & $7.9 \%$ & $1.0 \%$ \\
\hline Esp. >> Ptg. \{interrog. +$\}$ & $92.3 \%$ & $6.8 \%$ & $1.0 \%$ \\
\hline Esp. >> Ptg. \{otras categorías\} & $69.3 \%$ & $27.7 \%$ & $3.0 \%$ \\
\hline Portugués >> Español \{todos\} & $67.5 \%$ & $28.3 \%$ & $4.2 \%$ \\
\hline
\end{tabular}

Es evidente que, en la mayoría de los casos, las traducciones de oraciones mixtas se mantenían en una sola lengua, con una preferencia por el español, tanto en los estímulos español + portugués como en los enunciados portugués + español (tal vez, porque los participantes sabían que el investigador estudiaba el portugués de Misiones). Es especialmente notable el rechazo implícito de cambios después de palabras interrogativas, una configuración que suele impedir la alternancia de lenguas. De igual manera, no se mantenían los cambios de lengua después de un verbo auxiliar o un pronombre de sujeto, otros contextos desfavorecidos. Debido a la semejanza fonética entre los elementos negativos não (portugués) y no (español) en el habla natural, no era factible distinguir las palabras negativas con suficiente confiabilidad. Para la traducción de las oraciones mixtas, las diferencias entre las categorías llegaron a ser significativas. Un análisis de la varianza (ANOVA) realizado con las proporciones de retención de las configuraciones mixtas (sujetas a una transformación arcoseno) dio: $F_{1}(3,196)=3.36, p<.02 ; F_{2}(3,24)=3.17, p<.05$.

\section{SEGUNDO EXPERIMENTO: CLASIFICACIÓN DE LENGUAS}

Aunque todos los residentes de Misiones aceptan que el castellano y el brasilero (i.e. portugués) son lenguas distintas, muchos están convencidos de que en Misiones se habla "portuñol”, es decir alguna combinación improvisada de español y portugués. Por lo tanto, se llevó a cabo un experimento de clasificación para sondear los perímetros psicolingüísticos de las dos lenguas. El experimento se realizó en El Soberbio y Santa Rita.

\section{Participantes}

En este experimento participaron setenta y una personas bilingües (edades 18-50+) de las comunidades del Alto Uruguay (El Soberbio, Colonia Alicia, Santa Rita, Alba Posse y las colonias vecinas). 


\section{Materiales}

Se crearon 75 oraciones con voces sintetizadas: 20 en portugués, 15 en español, y 40 mixtas. Las oraciones mixtas contenían cambios de lengua entre pronombre de sujeto y verbo, entre verbo auxiliar e infinitivo, después de una palabra interrogativa inicial y en varias otras combinaciones.

\section{Método}

Por medio de una presentación programada en la plataforma PEBL (Mueller y Piper 2014) realizada en una computadora portátil, los participantes escuchaban los estímulos en orden aleatorio y clasificaban cada oración como español, portugués o mezclada. El programa recogía las respuestas y el tiempo de reacción. Para indicar la lengua, los participantes apretaban teclas señaladas con colores: mayúsculas (shift $\uparrow$ )-izquierda (rojo), espacio (azul) y mayúsculas (shift $\uparrow$ )-derecha (verde). En la pantalla aparecían imágenes que reforzaban la selección (véase la Gráfica 1).

Gráfica 1: Imágenes de pantalla para el experimento de clasificación

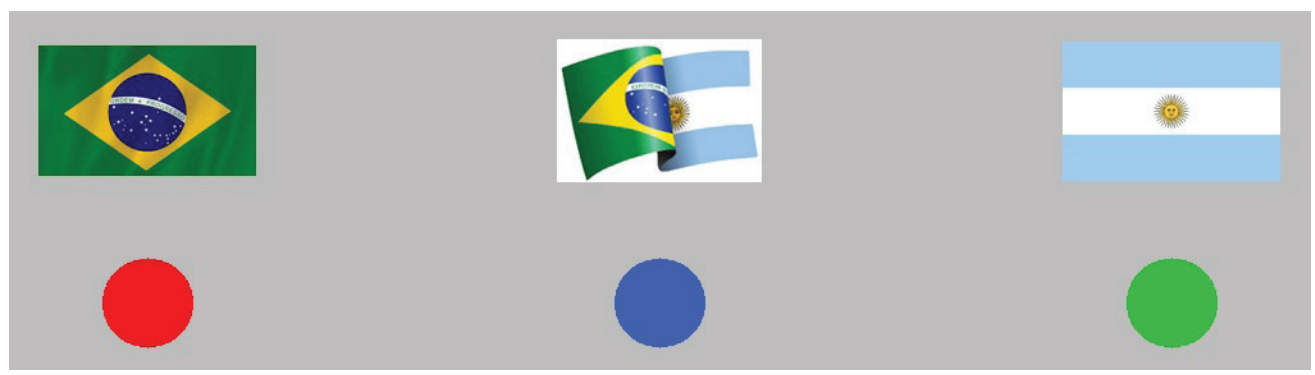

Resultados y discusión

Los resultados se presentan en los Cuadros 2 y 3 .

Cuadro 2: Resultados del experimento de clasificación $(\mathrm{N}=71)$

\begin{tabular}{|c|c|c|c|}
\hline & $\%$ clasificado esp. & $\%$ clasificado ptg. & \% clasificado mixto \\
\hline \multicolumn{4}{|l|}{ lengua del estímulo } \\
\hline español $(N=15)$ & $92.9 \%$ & $0.8 \%$ & $6.3 \%$ \\
\hline portugués $(\mathrm{N}=20)$ & $0.9 \%$ & $76.9 \%$ & $22.2 \%$ \\
\hline $\operatorname{mixto}(\mathrm{N}=45)$ & $4.5 \%$ & $33.6 \%$ & $61.9 \%$ \\
\hline
\end{tabular}


Cuadro 3: Resultados del experimento de clasificación; estímulos mixtos $(\mathrm{N}=71)$

\begin{tabular}{lc}
\hline & \% clasificado mixto \\
\hline tipo de mezcla & \\
\hline PRO + & $82.1 \%$ \\
Interrog. + & $58.2 \%$ \\
NEG + & $80.6 \%$ \\
aux+infinitivo & $83.6 \%$ \\
otras categorías & $75.7 \%$ \\
\hline
\end{tabular}

En el Cuadro 2 se ve que, aunque los participantes identificaban el español casi categóricamente, no manifestaban el mismo nivel de confianza al distinguir entre los enunciados en portugués y las oraciones mixtas, lo cual refleja el estatus extraoficial del portugués en Misiones y el alto grado de inseguridad que rodea el concepto popular de "portuñol". Las diferencias son significativas: $F_{1}(2,210)=118.7, p<.0001 ; F_{2}(2,72)=$ $12.8, p<.0001$. No obstante, esta ambigüedad el Cuadro 3 revela que los participantes reconocían como mixtas con una frecuencia aumentada las oraciones con cambio de lengua después de pronombres, negativos y verbos auxiliares. Al mismo tiempo, los cambios después de elementos interrogativos no resultaban en el mismo nivel de clasificaciones mixtas. Las diferencias en el reconocimiento de las distintas categorías mixtas eran significativas: $F_{1}$ (4, $350)=22.18, p<.0001 ; F_{2}(4,49)=10.03, p<.0001$.

\section{TERCER EXPERIMENTO: REPETICIÓN CON MEMORIA OPERATIVA SOBRECARGADA}

En el tercer experimento, los participantes tenían que repetir una oración después de haberse sobrecargado la memoria operativa con una distracción. Cuando se sobrecarga la memoria operativa, es posible que el participante no repita exactamente lo que escucha sino lo que piensa haber escuchado, es decir, lo que el participante mismo habría dicho (Gullberg, Indefrey y Muysken 2009: 34; Miller y Isard 1963; Marslen-Wilson1985; Vinther 2002). Este procedimiento ofrece una manera indirecta de sondear la competencia gramatical del participante sin pedir juicios explícitos de aceptabilidad, que en el caso de variedades lingüísticas no canónicas y personas de limitada formación escolar no son confiables.

\section{Participantes}

Participaron sesenta y siete de los participantes del experimento de clasificación, pero en otro momento. El experimento se llevó a cabo en El Soberbio y Santa Rita. 


\section{Materiales}

Para este experimento se prepararon veinte oraciones con voces sintetizadas, de las cuales ocho estaban en el portugués vernacular de Misiones y doce contenían combinaciones de portugués y español, con cambios de lengua después de pronombres de sujeto, palabras interrogativas, pronombres negativos y verbos auxiliares. Cada estímulo consistía en una oración seguida inmediatamente de un trozo de siete segundos de unos dibujos animados populares (Superman, Hombre Araña, Linterna Verde, Pájaro Loco, etc.). Después del video, aparecía una cuenta regresiva de diez segundos extraída de una película antigua. Finalmente aparecía la imagen de una boca. Se programó el experimento en PsychoPy (Pierce 2007), para presentar los estímulos en orden aleatorio.

\section{Método}

Para cada estímulo el participante debía retener en la memoria la oración que se escuchaba al comienzo. Durante la cuenta regresiva que seguía al dibujo animado, el participante describía el contenido del fragmento en el idioma que quisiera. Finalmente, al ver la imagen de la boca el participante debía repetir la oración original lo más exactamente posible. Los estímulos y las respuestas se grababan digitalmente. No se les revelaba a los participantes la existencia de estímulos que combinaban elementos del español y portugués.

\section{Resultados y discusión}

En el Cuadro 4 se presentan los resultados para los casos en que la respuesta consistía en la sustitución de elementos para lograr una oración monolingüe o la repetición sin alterar de la oración original.

Cuadro 4: Resultados de la repetición de enunciados mixtos español-portugués $(\mathrm{N}=67)$

\begin{tabular}{lccc}
\hline & $\%$ > $>$ Portugués & $\%$ > > Español & $\%$ sin alterar \\
\hline tipo de mezcla & & & \\
\hline mixto (todos) & $45.8 \%$ & $11.9 \%$ & $30.4 \%$ \\
PRO (esp.) + & $62.8 \%$ & $6.9 \%$ & $23.7 \%$ \\
Interrog. (esp.)+ & $25.5 \%$ & $4.0 \%$ & $40.2 \%$ \\
aux+infinitivo & $56.9 \%$ & $8.8 \%$ & $28.1 \%$ \\
otras categorías & $1.8 \%$ & $3.6 \%$ & $94.6 \%$ \\
\hline
\end{tabular}

Los niveles de "autocorrección" eran altos para los cambios después de pronombres y verbos auxiliares, lo cual confirma el estatus "especial" de estas configuraciones de alternancia. El nivel de corrección relativamente bajo para los interrogativos parece deberse 
a la semejanza fonética entre las palabras correspondientes en portugués y español. Por ejemplo, cuáles en espańol era repetido frecuentemente como cuallqual invariable (59.0\%). De igual manera, dónde mostraba un comportamiento ambiguo en cuanto a su base lingüística en las dos oraciones en que aparecía:

Dónde fica a casa do prefeito?

¿¿Dónde queda la casa del alcalde?'

Eu não sei dónde posso comprar um dicionário de inglês

'No sé dónde puedo comprar un diccionario de inglés'

En la primera oración, el $60.3 \%$ de las repuestas repetían la frase sin alteración, y otro $8.6 \%$ producían la palabra "inexistente" [dó.dzi], con palatalización de /d/ ante la vocal anterior (fenómeno muy extendido en el portugués brasileño, pero poco frecuente en el portugués de Misiones y las áreas brasileñas vecinas). Es posible que la variante arcaizante onde, que ocurre en el español rústico de muchas zonas, pueda haber borrado la frontera perceptual entre las dos lenguas. En la segunda oración, la posición intermedia de dónde resultaba en una tasa menor de repetición sin alteración (35.7\%), además de la variante dónde comprar (8.9\%), junto con el neologismo [dó.dzi] (14.3\%). En resumen, las diferencias de la retención de configuraciones mixtas entre categorías eran significativas: $\left.F_{1}(3,260)=41.35, p<.0001 ; F_{2}(3,14)=44.44, p<.0001\right)$, con importantes diferencias entre pronombres, interrogativas y auxiliares, con respecto a las otras categorías $(p<.0001)$, pero sin diferencias significativas entre las categorías de pronombre, interrogativo y auxiliar.

\section{DisCusión GENERAL}

La gran cantidad de elementos cognados, compartidos por el español y el portugués, facilita la compenetración de lenguas en el entorno sociolingüístico de Misiones, aun cuando los hablantes no suelen mezclar las lenguas conscientemente. En los experimentos, los participantes detectaban configuraciones mezcladas, y en las tareas de repetición y traducción, las enmendaban para lograr enunciados monolingües. Aunque no todas las mezclas fueron reconocidas implícita o explícitamente, los participantes rechazaban muchos de los cambios de lengua después de pronombres y verbos auxiliares con una frecuencia más alta, lo cual coincide con las observaciones sobre contactos entre lenguas tipológicamente más variadas (p. ej., español-inglés). Como consecuencia de la semejanza fonética entre los elementos negativos (no en español y não en portugués), no todos los cambios entre palabra negativa y verbo eran detectados por los participantes, de manera que el estatus de aquella configuración queda sin resolver. Las palabras interrogativas desplazadas al comienzo de la oración no provocaban el mismo nivel de rechazo, a pesar de que esta configuración ha sido señalada como problemática en otras investigaciones de los cambios de código. Al igual que 
la negación, la semejanza fonética entre las palabras correspondientes en español y portugués (p. ej. dónde - onde, quién - quem, cuáles - quais) puede contribuir a la baja perceptibilidad de los cambios de lengua alrededor de los interrogativos.

Los datos de la interfaz portugués-español en Misiones demuestran la complejidad psicolingüística de la alternancia de lenguas, tratándose en este caso de dos lenguas genealógicamente cercanas cuyas historias se han entretejido durantelargos años, sobre todo en zonas fronterizas. Entre el español y el portugués las postuladas restricciones morfosintácticas sobre los cambios de código intra-oracionales no son ni completamente inexistentes ni totalmente vigentes sino variablemente franqueables, lo cual sugiere una relación estrecha entre repertorio léxico/morfosintáctico y configuraciones mixtas: un umbral basado en la distancia psicolingüística entre las lenguas, más allá del cual se debilitan o desaparecen las restricciones gramaticales sobre los cambios intra-oracionales. La cercanía tipológica y léxica entre las lenguas puede facilitar la lexicalización congruente definida por Muysken (2000), es decir la compenetración casi libre de lenguas cuyas estructuras morfosintácticas y léxicas son muy similares, y es posible que el umbral psicolingüístico que define las restricciones intra-oracionales se incorpore a la tipología bilingüe como característica fundamental de la lexicalización congruente. El trabajo que se acaba de presentar representa un primer intento de aplicar métodos psicolingüísticos al estudio del bilingüismo castellano-portugués, para profundizar los conocimientos sobre la relación entre tipología lingüística y alternancia de lenguas. En la medida en que los resultados sean representativos de las respectivas comunidades de habla, la permeabilidad de las restricciones morfosintácticas puede servir como elemento diagnóstico en los estudios tipológicos, con la hipótesis de una correlación inversa entre distancia genealógica y aceptabilidad de algunas combinaciones bilingües. Las técnicas experimentales complementan las investigaciones variacionistas realizadas hasta la fecha e incorporan un llamado para la colaboración interdisciplinaria para avanzar en el estudio del bilingüismo.

\section{Obras citadas}

Aguazo, Cristian y Clarice N. von Borstel. 2006. Estudos sociolinguísticos da comunidade de fala de Vila Velha, Guaíra, Paraná. Relatório de Iniciação de Pesquisa do PIBIC/ CNPq/Unioeste/2006, 1-15.

Amable, Hugo. 1975. Las figuras del habla misionera. Santa Fe: Librería y Editorial Colmegna.

Azuma, Shoji y Meier, Richard. 1997. Open class and closed class: sentence-imitation experiments on intrasential code-switching. Applied Psycholinguistics 18. 257-276.

Belazi, Heidi, Edward Rubin, y Almeida Jacqueline Toribio. 1994. Code-switching and Xbar theory: the Functional Head Constraint. Linguistic Inquiry 25. 221-238.

Bhatt, Rakesh Mohan. 1997. Code-switching, constraints, and optimal grammars. Lingua 102.223-251. 
Boersma, Paul y D.Weenink. 1999-2005. PRAAT: Doing phonetics by computer. http:// www.fon.hum.uva.nl/praat/

Borstel, Clarice Nadir von. 2011. O code switching sobre a visão do modelo variacionista. UniLetras 23. 147-155.

- - -. 2011. As situações enunciativas de alternância de códigos em línguas de fronteiras. Linguas em contato: cenários de bilinguísmos no Brasil, ed. Sidney de Souza Silva, 309333. Campinas: Pontes Editores.

Bullock, Barbara y Almeida Jacqueline Toribio (eds.). 2009. The Cambridge handbook of linguistic code-switching. Cambridge: Cambridge University Press.

Carvalho, Ana Maria. 2003a. Rumo a uma definição do Português Uruguaio". Revista Internacional de Lingüistica Iberoamericana 1(2). 125-149.

- - -. 2003b. The sociolinguistic distribution of (lh) in Uruguyan Portuguese: a case of dialect diffusion". Linguistic theory and language development in Hispanic languages, ed. Silvina Montrul y Francisco Ordóńez. 30-44. Somereville, MA: Cascadilla Press.

- - - 2004a. I speak like the guys on TV: palatalization and the urbanization of Uruguayan Portuguese. Language Variation and Change 16. 127-151.

- - - 2004b. Diagnóstico sociolingüístico de comunidades escolares fronterizas en el norte de Uruguay". Portugués del Uruguay y educación bilingüe, ed. Claudia Brovetto y Javier Geymonat, 44-96. Montevideo: Administración Nacional de Educación Pública.

- - - 2006. Políticas lingüísticas de séculos passados nos dias de hoje: o dilema sobre a educação bilíngüe no norte do Uruguai". Language Problems and Language Planning 30. 149-171.

Chan, Brian Hok-Shing. 2009. Code-switching between typologically distinct languages. The Cambridge handbook of linguistic code-switching, ed. Barbara Bullock y Almeida Jacqueline Toribio, 182-198. Cambridge: Cambridge University Press.

Clyne, Michael. 1987. Constraints on code switching: how universal are they? Linguistics 25. 739-764.

Daviña, Liliana Silvia. 2003. Fronteras discursivas en una región plurilingüe: español y portugués en Misiones. Tesis de maestría, Universidad de Buenos Aires.

Deuchar, Margaret, Pieter Muysken y Sung-Lan Wang. 2007. Structured variation in codeswitching: towards an empirically based typology of bilingual speech patterns. International Journal of Bilingual Education and Bilingualism 10. 298-340.

DiSciullo, Anne-Marie, Pieter Muysken, y Rajendra Singh. 1986. Government and codemixing. Journal of Linguistics 22. 1-24.

Doron, Edit. 1983. On a formal model of code-switching. Texas Linguistic Forum 22. 3559.

Douglas, Kendra. 2004. Uruguayan Portuguese in Artigas: tri-dimensionality of transitional local varieties in contact with Spanish and Portuguese standards. Tesis doctoral, University of Wisconsin.

Dussias, Paola. 2003. Spanish-English code mixing at the Auxiliary Phrase: evidence from 
eye-movement data". Revista Internacional de Lingüistica Iberoamericana 1(2). 7-34.

Ebert, Shane. 2014. The morphosyntax of Wh-questions: evidence from Spanish-English code-switching. Tesis doctoral, University of Illinois, Chicago.

Elizaincín, Adolfo. 1973. Algunos aspectos de la sociolingüistica del dialecto fronterizo. Montevideo: Universidad de la República.

- - -. 1976. The emergence of bilingual dialects on the Brazilian-Uruguayan border". International Journal of the Sociology of Language 9. 123-134.

- - - 1979. Algunas precisiones sobre los dialectos portugueses en el Uruguay. Montevideo: Universidad de la República.

- - -. 1992. Dialectos en contacto: español y portugués en España y América. Montevideo: Arca.

Elizaincín, Adolfo y Luis Behares. 1981. "Variabilidad morfosintáctica de los dialectos portugueses del Uruguay”. Boletín de Filología 31 .401-417.

Elizaincín, Adolfo, Luis Behares y Graciela Barrios. 1987. Nos falemo brasilero. Montevideo: Editorial Amesur.

Gómez Rendón, Jorge. 2008. Mestizaje lingüístico en los Andes: génesis y estructura de una lengua mixta. Quito: Abya-Yala.

Gullberg, Marianne, Peter Indefrey y Pieter Muysken. 2009. Research techniques for the study of code-switching. The Cambridge handbook of linguistic code-switching, ed. Barbara Bullock y Almeida Jacqueline Toribio, 21-39. Cambridge: Cambridge University Press.

Gumperz, John. 1977. The sociolinguistic significance of conversational code-switching. RELC Journal 8.1-34.

Hebblethwaite, Benjamin. 2007. Intrasential code-switching among Miami Haitian Creole-English bilinguals. Tesis doctoral, Indiana University.

Hensey, Fritz. 1972. The sociolinguistics of the Brazilian-Portuguese border. La Haya: Mouton.

- -. 1975. Fronterizo: a case of phonological restructuring". Three essays on linguistic diversity in the Spanish-speaking world, ed. Jacob Ornstein, 47-59. Rowley, MA: Newbury House.

- - . 1982a. Uruguayan fronterizo: a linguistic sampler". Word 33. 193-198.

- - - 1982b. Spanish, Portuguese and Fronteriço: languages in contact in northern Uruguay". International Journal of the Sociology of Language 34. 9-23.

Hogeweg, Liselot. 2009. Word in process: On the interpretation, acquisition, and production of words. Tesis doctoral, Radboud University Nijmegen, Holanda.

Jake, Janet, Carol Myers-Scotton y Steven Gross. 2002. Making a minimalist approach to codeswitching work: adding the Matrix Language. Bilingualism: language and cognition 5. 69-91.

Joshi, Aravind. 1985. Processing of sentences with intrasentential code switching. Natural language parsing: psychological, computational and theoretical perspectives, ed. David Dowty, Lauri Karttunen y Arnold Zwicky, 190-205. Cambridge: Cambridge University Press. 
Kaufmann, Göz. 2009. Falar espanhol or hablar portugués: attitudes and linguistic behavior on the Brazilian-Uruguayan and Brazilian-Argentinian borders. Romanistisches Jahrbuch 60. 276-317.

Koronkiewicz, Bryan. 2014. Pronoun categorization: evidence from Spanish/English codeswitching. Tesis doctoral, University of Illinois at Chicago.

Lipski, John. 1977. Bilingual code-switching and linguistic competence. Fourth LACUS Forum, 263-277. Columbia, SC: Hornbeam Press.

- - . 1978. Bilingual language switching and the problem of linguistic competence. Aspects of bilingualism, ed. Michel Paradis, 250-264. Columbia, SC: Hornbeam Press.

- - . 1982. SpanishEnglish language switching in speech and literature: theories and models. Bilingual Review 9. 191-212.

- - . 1985. Linguistic aspects of SpanishEnglish language switching. Tempe: Arizona State University, Center for Latin American Studies.

- - - 2006. Too close for comfort? the genesis of "portuñol/portunhol. Selected proceedings of the 8th Hispanic Linguistics Symposium, ed. Timothy L. Face y Carol A. Klee, 1-22. Somerville, MA: Cascadilla Press. Disponible en: www.lingref.com/cpp/wss/2/ paper1136.pdf

- - - 2008. Searching for the origins of Uruguayan Fronterizo dialects: radical code-mixing as ‘fluent dysfluency’. Journal of Portuguese Linguistics 8. 5-46.

- - -. 2009. 'Fluent dysfluency' as congruent lexicalization: a special case of radical codemixing”. Journal of Language Contact 2. 1-39.

- - . 2010. Spanish and Portuguese in contact. Handbook of language contact, ed. Raymond Hickey, 550-580. Nueva York: Wiley-Blackwell.

- - - 2011a. Um caso de contato de fronteira: o sudoeste. Os contatos linguísticos no Brasil, ed. Heliana Mello, Cléo Altenhofen y Tommaso Raso, 349-368. Belo Horizonte: Editora UFMG.

- - . 2011b. Encontros lingüísticos fronteiriços. Ideação 13(2). 83-100. Disponible en http://e-revista.unioeste.br/index.php/ideacao/article/viewArticle/6109

- - -. 2014. Spanish-English code-switching among semi-fluent bilinguals: towards an expanded typology. Sociolinguistic Studies 8. 23-55.

- - . 2015. Portuguese/Portuñol in Misiones, Argentina: another "Fronterizo"? New perspectives on Hispanic contact linguistics in the Americas, ed. Sandro Sessarego y Melvin González, pp. 253-281. Frankfurt y Madrid: Vervuert/Iberoamericana.

- - . 2016a. Language switching constraints: more than syntax? Data from Media Lengua. Bilingualism: Language and Cognition doi:10.1017/S1366728916000468

- - -. 2016b. The role of unintentional/involuntary code-switching: Did I really say that? Spanish-English Codeswitching in the Caribbean and the US, ed. Rosa Guzzardo Tamargo, Catherine M. Mazak y M. Carmen Parafita Couto, 139-168. Amsterdam: John Benjamins.

- - . 2017. Portuguese or Portuñol? Language contact in Misiones, Argentina. Journal of Linguistic Geography 4. 1-18. 
MacSwan, Jeff. 1999. A minimalist approach to intrasentential code switching. Nueva York: Garland Press.

- - - 2000. The architecture of the bilingual language faculty: evidence from intrasentential code switching. Bilingualism: language and cognition 3. 37-54.

- - -. 2004. Codeswitching and linguistic theory". Handbook of bilingualism, ed. Tej Bhatia y William Ritchie, 283-310. Malden, MA y Oxford: Blackwell.

- - - 2005. Codeswitching and generative grammar: a critique of the MLF model and some remarks on 'modified minimalism'. Bilingualism: language and cognition 8. 1-22.

Mahootian, Shahrzad y Santorini, Beatrice. 1996. Code switching and the complement/ adjunct distinction. Linguistic Inquiry 27. 464-479.

Maia, Ivene Carissini da. 2004. Intercambios lingüísticos de frontera: incidencia en el hablar de los alumnos del profesorado en portugués de la UNAM. Tesis de maestría, Universidad Nacional de Misiones.

Marslen-Wilson, William. 1985. Speech shadowing and speech comprehension. Speech Communication 4. 55-73.

Miller, George y Stephen Isard. 1963. Some perceptual consequences of linguistic rules. Journal of Verbal Learning and Verbal Behavior 2. 217-228.

Moyer, Melissa. 1992. Analysis of code-switching in Gibraltar. Tesis doctoral, Universitat Autònoma de Barcelona.

Mueller, Shane T. y Piper, Brian J. 2014. The Psychology Experiment Building Language (PEBL) Test Battery. Journal of Neuroscience Methods 222. 250-259.

Muysken, Pieter. 1979. La mezcla de quechua y castellano: el caso de la "media lengua" en el Ecuador. Lexis 3. 41-56.

-. -. 1981. Halfway between Quechua and Spanish: the case for relexification. Theoretical orientations in creole studies, ed. Albert Valdman y Arnold Highfield, 52-78. Nueva York: Academic Press.

- - . 2000. Bilingual speech: a typology of code-mixing. Cambridge: Cambridge University Press.

- - - 2013. Language contact outcomes as the result of bilingual optimization strategies. Bilingualism: Language and Cognition 16. 709-730.

Myers-Scotton, Carol. 1997. Dueling languages: grammatical structure in codeswitching. Oxford and New York: Oxford University Press, $2^{\mathrm{a}}$ ed.

Peñalosa, Fernando. 1980. Chicano sociolinguistics: a brief introduction. Rowley, MA: Newbury House.

Peirce, Jonathan W. 2007. PsychoPy - Psychophysics software in Python. Journal of Neuroscience Methods 162 (1-2). 8-13. El programa está disponible en: www.psychopy.org

Pfaff, Carol. 1979. Constraints on language mixing: intrasentential code-switching and borrowing in Spanish/English. Language 55. 291-318.

Poplack, Shana. 1980. Sometimes I'll start a sentence in Spanish y termino en español. Linguistics 18. 581-618.

Prince, Elle y Pintzuk, Susan. 2000 [1983]. Bilingual code-switching and the open/closed class distinction. University of Pennsylvania Working Papers in Linguistics (PWPL) 
6(3).237-257.

Rona, José Pedro. 1960. La frontera lingüística entre el portugués y el español en el norte del Uruguay. Veritas 8. 201-219.

- - . 1969. El dialecto "fronterizo" del norte del Uruguay. Montevideo: Adolfo Linardi

Semino, Maria Josefina Israel y Patrícia Mussi Escobar. 2011. Las interferencias en la frontera Uruguay-Brasil: un estudio de caso sobre las restricciones. Linguas em contato: cenários de bilinguísmos no Brasil, ed. Sidney de Souza Silva, 335-350. Campinas: Pontes Editores.

Sturza, Eliana Rosa. 2005. Línguas de fronteira: o desconhecido território das práticas lingüisticas nas fronteiras. Ciência e Cultura 57(2). 47-50. Disponible en http://cienciaecultura.bvs.br/pdf/cic/v57n2/a21v57n2.pdf

Timm, Leonora. 1975. Spanish-English code-switching: el porque y how not to. Romance Philology 28. 473-482.

Toribio, Almeida Jacqueline. 2001a. Accessing billingual code-switching competence. International Journal of Bilingualism 5. 403-436.

- - - 2001b. On the emergence of bilingual code-mixing competence. Bilingualism: language and cognition 4. 203-231.

Van Gelderen, Elly y Jeff MacSwan. 2008. Interface conditions and code-switching: an Fmovement analysis of pronouns and lexical DPs. Lingua 118. 765-776.

Vinther, Thora. 2002. Elicited imitation: a brief overview. International Journal of Applied Linguistics 12. 54-73.

Woolford, Ellen. 1983. Bilingual code-switching and syntactic theory. Linguistic Inquiry 13.519-535.

-. -. 1984. On the application of WH movement and inversion in code-switching sentences. Revue Quebecoise de Linguistique 14. 77-86. 